\title{
Age at Full-Time Use
}

National Cancer Institute

\section{Source}

National Cancer Institute. Age at Full-Time Use. NCI Thesaurus. Code C139175.

The age at which the full-time use of an entity occurred. 